\title{
Thermal analysis of the fused filament fabrication printing process: Experimental and numerical investigations
}

\author{
David Xu · Yancheng Zhang · Franck Pigeonneau
}

Received: date / Accepted: date

\begin{abstract}
A 3d printing of a thin wall is achieved by a fused filament fabrication process. The influence of the printing velocities on the filament morphology is studied using optical microscopy. The strand morphology is approximated to different geometries and compared to experimental data. The oblong cross-section is a good approximation to estimate the strand's height and width. A local temperature is recorded by introducing a thermocouple during the printing of a thin wall. The polymer undergoes successive heating and cooling. Their magnitudes decrease with time while the filament deposition occurs farther from the thermocouple location. A steady-state cooling is observed after an extended period of time due to the surrounding air cooling. The influence of the strand's cross-section area on its cooling kinetics is investigated experimentally.

The printing of a thin wall with the same geometry is also numerically computed by solving the heat transfer equation with a finite element method. The thermal conductivity takes into account the porosity of the printed wall. An estimation of the heat transfer coefficients between the wall and the surrounding air is done by comparison with a particular experiment. The numerical computation reproduces very well the amplitudes and the periods of heating and cooling observed experimentally. Moreover, the changes in the morphology of the melted filament show the reliability of the numerical tool to obtain a thermal history in agreement with experimental data.
\end{abstract}

Keywords $3 \mathrm{~d}$ printing $\cdot$ material extrusion $\cdot$ amorphous polymer $\cdot$ heat transfer $\cdot$ strand morphology $\cdot$ finite element analysis

D. Xu

MINES ParisTech, PSL Research University, CEMEF - Centre for materials forming, CNRS UMR 7635, CS

10207, rue Claude Daunesse 06904 Sophia Antipolis Cedex, France

E-mail: david.xu@mines-paristech.fr

Y. Zhang

MINES ParisTech, PSL Research University, CEMEF - Centre for materials forming, CNRS UMR 7635, CS

10207, rue Claude Daunesse 06904 Sophia Antipolis Cedex, France

E-mail: yancheng.zhang@mines-paristech.fr

F. Pigeonneau

MINES ParisTech, PSL Research University, CEMEF - Centre for materials forming, CNRS UMR 7635, CS

10207, rue Claude Daunesse 06904 Sophia Antipolis Cedex, France

E-mail: franck.pigeonneau@mines-paristech.fr 


\section{Introduction}

Additive manufacturing (AM) is a widely growing technology due to its ability to build objects with complex shapes. Moreover, this process minimises post-processing and reduces material wastes. Different materials can be printed such as metals, ceramics and polymers [4]. Techniques such as laser-based processes, extrusion processes, material jetting, adhesive or electron beams are being developed.

The Fused Filament Fabrication (FFF) process is based on the deposition of melted polymer filaments to create an object from a 3d CAD model [8]. This process is widely used since it is easy to operate, reproducible, low cost and can accept sustainable materials [7]. According to Goh et al. [8], the melting and solidification of thermoplastics is an open problem due to fast heating and cooling during the printing. The heating of a polymer in the liquefier has been recently addressed by MacKay et al. [12] who determined the maximum velocity for a given temperature field at the nozzle exit. Peng et al. [15] achieved experiments by introducing a thermocouple in the filament to record the temperature of the polymer during the residence through the liquefier. Pigeonneau et al. [16] performed numerical computations of the thermal behaviour inside a liquefier. They showed that this stage is similar to the so-called Graetz problem [19]. Criteria can be determined to control the melting of the extruded material. The solidification has been addressed by McIlroy and Olmsted [14] for amorphous polymers. McIlroy and Graham [13] extended this work for semi-crystalline polymers. Isothermal flow deposition of an amorphous polymer has been simulated by Comminal et al. [5]. The model predicts the deposited strand morphology based on the printing velocities, the gap and the liquefier exit diameter. The developed model is experimentally assessed by Serdeczny et al. [18].

The bonding quality between the solidified strands is a key parameter to print products with good mechanical properties [22]. Indeed, the failures are most generally localised on the welding areas [17]. Yang and Pitchumani [23] developed a criterion to measure the interdiffusion efficiency of polymer chains at the contact between two polymer layers. A degree of healing is then defined as the ratio of the interfacial bond strength to the ultimate bond strength.

Based on a simplified model, Bellehumeur et al. [3] developed an analytic solution to describe the cooling of a single deposited strand. A sintering model controlled by the material viscosity and surface tension was developed to predict bond formation. Sun et al. [20] have experimentally evaluated this model by measuring the temperature at the bottom layer of a printed part and by measuring the variation in the neck growth between adjacent filaments. The theoretical model underestimates neck growth between adjacent strands.

Costa et al. [6] studied the different heat transfer modes involved in the cooling of polymer filaments. The cooling is mainly due to the thermal conduction between adjacent strands and with the substrate. Moreover, the convective heat transfer with the surrounding air contributes to the cooling. Costa et al. [7] developed a numerical cooling model taking into account all physical contacts between the strands throughout the whole process. The cross-sectional average temperature is computed as a function of time. Experimental measurements were also performed by a thermography set-up. A good agreement between simulations and experiments is observed for the first three stacked polymer filaments.

Zhang and Shapiro [25] computed the temperature field in a FFF printed product using a finite-difference method. Multiple contacts between printed strands are taken into account. The model thus takes into account the different heat transfer phenomena corresponding to these contacts. Thermal computations are only performed on segments directly influenced 
by the deposition of a new segment. The overall temperature is predicted with minimum computation time.

The strand morphology is an important information to calibrate the deposition path during the printing. Therefore, in the present study, experimental measurements are done to estimate the strand geometry as a function of printing parameters. The knowledge of the temperature field over time is a critical information to predict the bonding quality between strands and thus mechanical performances of the printed object. Therefore, the thermal behaviour during printing is experimentally determined. The influence of the strand shape on the cooling kinetics is investigated. Numerical computations are achieved by solving the heat transfer equation and assessed by experimental measurements. The major issue addressed here is to present a numerical tool enable to accurately predict the temperature field during the printing. The knowledge of the temperature behaviour when printing an object is the first step to investigate and enhance the bonding quality of a final product.

In section 2, material and experimental setup are detailed. Section 3 describes the numerical model to determine the temperature field. Experimental results are presented in $\S 4$. Numerical results and comparison with the experimental measurements are provided in sections 5. Conclusions are drawn in section 6.

\section{Experimental procedure}

\subsection{Material and experimental setup}

The amorphous polymer used in this study is a commercial-grade acrylonitrile butadiene styrene (ABS) polymer sold by the brand Grossiste3D. The ABS polymer is chosen in this study since it is a very common amorphous polymer with little dependence on temperature of its density and specific heat capacity. It is thus easier to focus our study on its thermal behaviour. The glass transition temperature of the polymer has been measured by a DSC 4000 from PerkinElmer ${ }^{\circledR}$ with a cycle of $30-300^{\circ} \mathrm{C}$. The heating rate is set at $50 \mathrm{~K} \mathrm{~min}^{-1}$. The glass transition temperature $T_{g}$ is equal to $110^{\circ} \mathrm{C}$ which is the typical value for this polymer. The thermal properties of the $\mathrm{ABS}$ polymer required for numerical computations are given in Table 1 .

The $3 \mathrm{~d}$ printer employed in these experiments is a Delta turbo 2040 from WASP ${ }^{\circledR}$. The temperature is measured using T-type thermocouples and a midi GL220 from Graphitec ${ }^{\circledR}$ as the data logger.

\subsection{The Fused Filament Fabrication process}

The FFF process consists of feeding a liquefier with a solid filament polymer via a pinch roller mechanism as sketched in Fig. 1. The polymer is heated above its melting temperature in the liquefier. The melted filament is then deposited on the build surface where it cools down and solidifies. Our liquefier's exit diameter $D$ is equal to $400 \mu \mathrm{m}$. The build surface is heated for processing purposes. Different cooling kinetics are observed during the printing process. Consequently, heterogeneous temperature distribution appears through the printed object. The gap between the exit of the liquefier and the last deposited layer $g$ (see Fig. 1) is constant during the printing. The deposition path, as well as the extrusion and substrate temperatures are to be specified. These parameters are set in a G-code file used to drive the $3 d$ printer. 


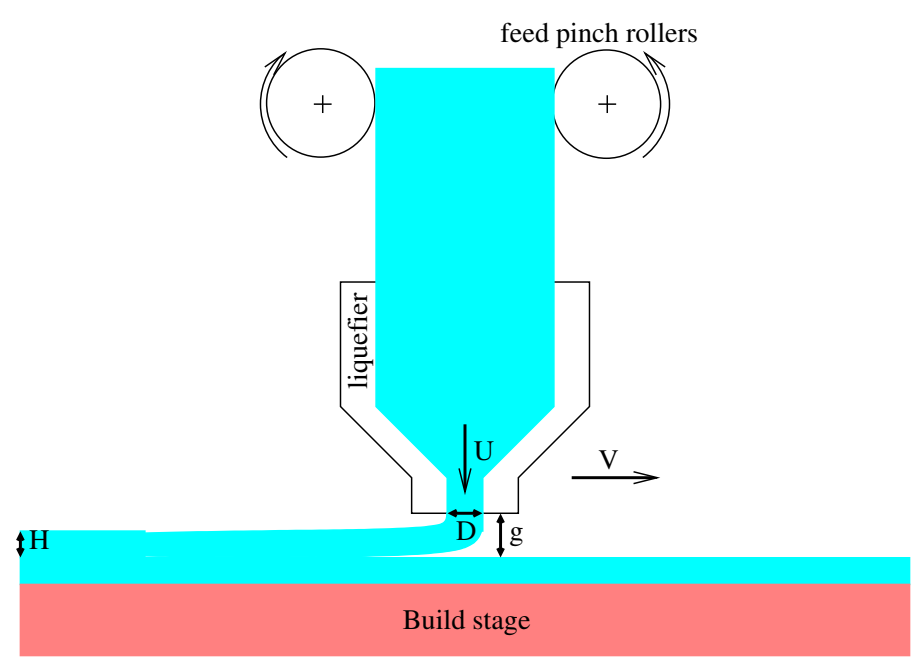

Fig. 1: A sketch of the FFF process with the main printing parameters.

\subsection{Deposited strand morphology}

The size of the deposited polymer filament is mainly driven by the extrusion velocity $U$ and the nozzle velocity $V[5,1]$. Fig. 1 represents a sketch of the FFF process in which $U$ and $V$ are reported. The size of the deposited strand is an important data to set the deposition path of the nozzle. Indeed, the printing gap $g$ is set as a function of the strand height $H$. The printing distance between strands depends on strand's width $W$. The strand's height and width are thus measured with an optical microscope Olympus ${ }^{\circledR}$ PMG3 in reflection mode. Single strands are printed at different printing velocities $U$ and $V$. The strands are then cut at one half of their length. Images of the strand cross section are captured using the optical microscope as illustrated in Fig. 2. The left picture corresponds to the case with an extrusion velocity $U=6 \mathrm{~m} \mathrm{~min}^{-1}$ and $V=2 \mathrm{~m} \mathrm{~min}^{-1}$ while the right picture is obtained with $U=4 \mathrm{~m} \mathrm{~min}^{-1}$ and $V=2 \mathrm{~m} \mathrm{~min}^{-1}$. Measurements are done using the ARCHIMED software from Microvision Instruments. The gap and nozzle diameter stay constant to know the influence of the printing parameters $U$ and $V$ on strand morphology.

\subsection{Procedure of the wall printing}

A wall with a length $L$ and a height $H$ set to 10 and $1 \mathrm{~cm}$ respectively is printed with different printing velocities. The wall is composed of four polymer filaments meaning that its thickness depends on the printing velocities. The thermal behaviour depending on the printing conditions is studied during the printing of the wall. The temperature is recorded using T-type thermocouples. This technique is chosen instead of the infrared thermography since a thermocouple allows to directly measure the temperature inside a layer. Moreover, few tries using infrared thermography have shown that measurements are disturbed by thermal radiation of the printer's hot-end. The T-type thermocouple is introduced during a printing pause of eight seconds when the wall height is equal to $5 \mathrm{~mm}$. The knowledge of the exact position of the thermocouple is a fastidious task as it is impossible to put markers on the printed 
(a) $U=6 \mathrm{~m} \mathrm{~min}^{-1}$ and $V=2 \mathrm{~m} \mathrm{~min}^{-1}$.

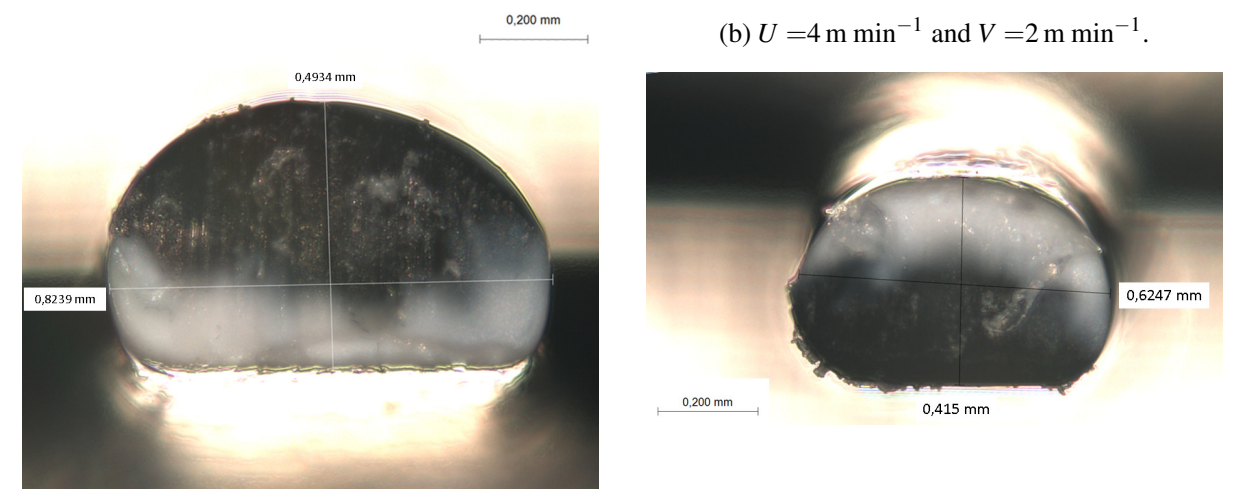

Fig. 2: Examples of strand's dimension measurements.

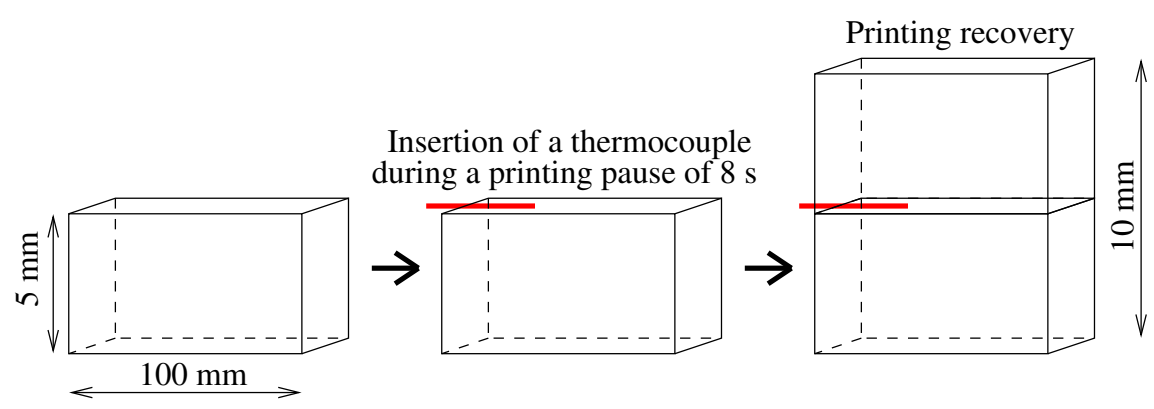

Fig. 3: Side view of the three steps of the thermocouple insertion during the wall printing composed by four filaments.

object. However, the thermocouple is always introduced during the printing of the second line, at one-third of $L$. Fig. 3 illustrates the printing experiment with the wall just before the introduction of the thermocouple on the left. The insertion of the thermocouple is depicted in the central sketch. The right picture represents the wall at the end of the experiment.

The location of the thermocouple in the inserted layer is illustrated in Fig. 4. The arrows depict the printing direction of the nozzle. The same deposition path is reproduced for all tested conditions. For each tested condition, a minimum of four experiments are achieved. An average of all data is then computed to know the average temperature behaviour for a particular set of parameters.

Fig. 5 depicts the temperature recording of five experiments achieved with $U=4 \mathrm{~m} \mathrm{~min}^{-1}$ and $V=1 \mathrm{~m} \mathrm{~min}^{-1}$. Despite uncertainties on the accurate location of the thermocouple, the reproducibility is satisfying.

\section{Numerical finite element model}

Thermal numerical computations are performed based on the model developed by Zhang et al. [24] and implemented in the $\mathrm{C}++$ library CimLib. Initially developed for the selec- 


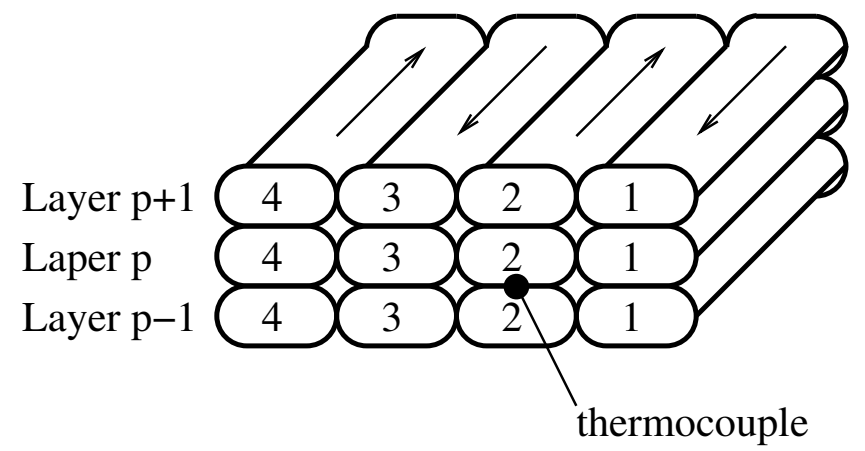

Fig. 4: Scheme of the section of a wall where the thermocouple is placed. The arrows depict the printing direction of the nozzle.

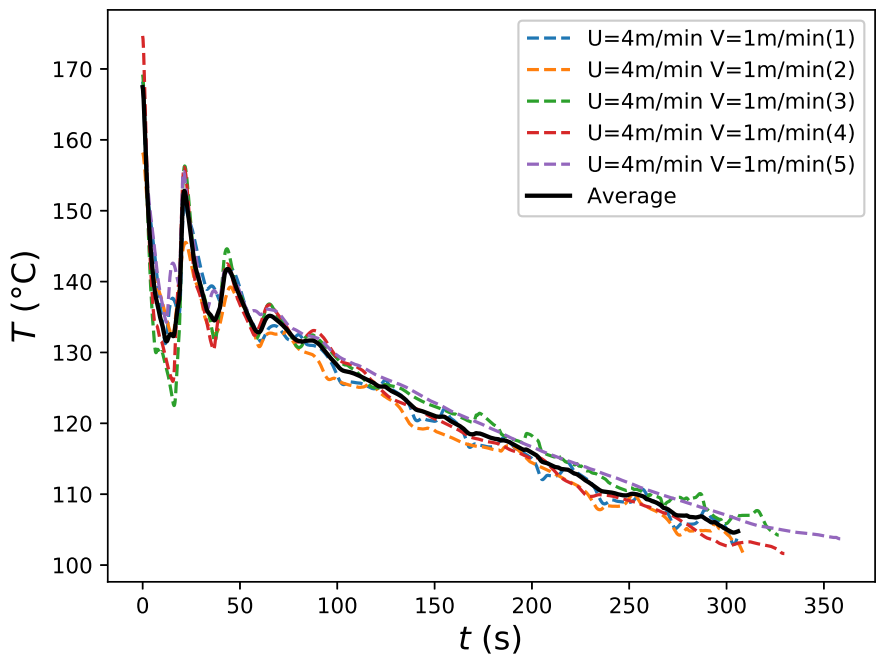

Fig. 5: Example of the average temperature evolution for a set of printing parameters.

tive laser melting process, the numerical procedure is thus modified to match with the FFF process.

\subsection{Numerical procedure to print a wall}

A rectangular domain of width $W$, length $L$ and height $H$ similar to the experiments presented in the previous section is numerically printed. The computational domain is initially composed of air. The polymer is deposited by the introduction of a series of fractions within one layer. The deposition sequence is based on a G-code file. Table 1 gives the thermal properties of the ABS polymer according to Costa et al. [7]. The melted polymer is deposited on a meshed substrate made of steel with thermal properties given in Table 1. 


\begin{tabular}{ccccc}
\hline Properties & Notation & Unit & ABS & Steel \\
\hline Density & $\rho$ & $\mathrm{kg} / \mathrm{m}^{3}$ & 1050 & 7850 \\
Specific heat & $C_{p}$ & $\mathrm{~J} \mathrm{~kg}^{-1} \mathrm{~K}^{-1}$ & 2200 & 477 \\
Thermal conductivity & $k$ & $\mathrm{~W} \mathrm{~m}^{-1} \mathrm{~K}^{-1}$ & 0.18 & 42.7 \\
\hline
\end{tabular}

Table 1: Properties of the ABS polymer according to Costa et al. [7] and of an AISI 4130 steel.

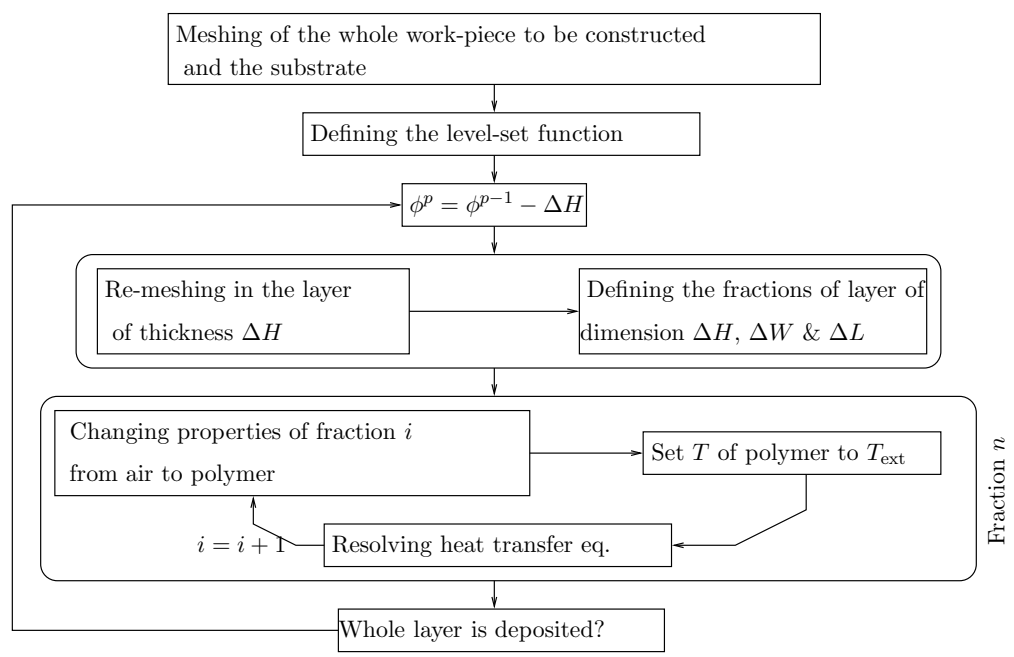

Fig. 6: Flow chart of the different steps of the numerical computation of the wall.

The flow chart of the numerical computation of a wall is summarised in Fig. 6. A levelset function $\phi$ is used to define the building front of the deposited polymer. Each layer has a given height $\Delta H$. The zero value of $\phi$ corresponds to the position $z=H$ of the deposited strand. The level-set function $\phi$ is thus reinitialised at each layer increment $p$ as follows

$$
\phi^{p}=\phi^{p-1}-\Delta H
$$

Re-meshing is performed before the deposition of the next layer.

The wall width $W$ is divided into lines of same size $\Delta W$ representing strands of polymer. A single strand of length $L$ is divided into fractions of length $\Delta L$. The geometry of the strand is assumed rectangular in the numerical computation. Each fraction of strand is successively added after a time step dependent on the nozzle velocity $V$. A single strand line is deposited during a time $t_{l}$ defined as follows

$$
t_{l}=\frac{L}{V} .
$$

The deposition time of a strand fraction $t_{f}$ depends on the number of fractions $n$ of a strand defined by

$$
t_{f}=\frac{t_{l}}{n}=\frac{\Delta L}{V} .
$$

After each time step $t_{f}$, thermal properties of a volume fraction $\Delta H \Delta W \Delta L$ change from air to polymer. The successive deposition of melted polymer during $3 \mathrm{~d}$ printing is thus mimicked. 
(a) Deposition of a sub-layer at $t=n t_{f}$

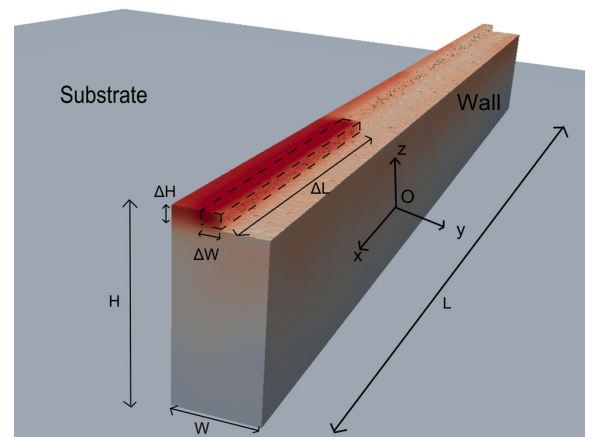

(b) Deposition of a sub-layer at $t=(n+1) t_{f}$

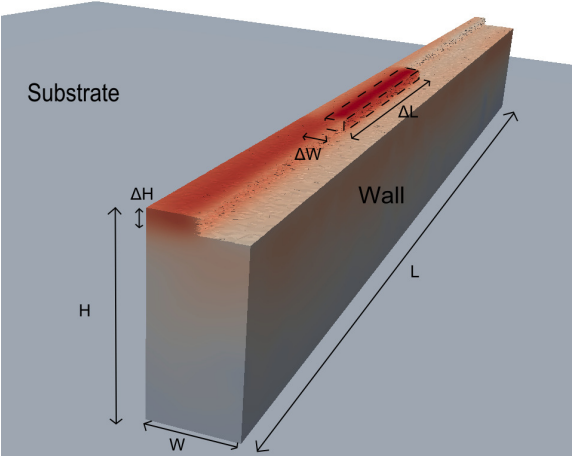

Fig. 7: Numerical printing of a wall with two steps of the printing to simulate the FFF process. The origin and the Cartesian coordinate system is depicted in sub-figure 7a.

The numerical procedure is illustrated in Fig. 7. Sub-figure 7a is the state of deposition at the time $n t_{f}$ while sub-figure $7 \mathrm{~b}$ represents the state at the time $(n+1) t_{f}$. Dimensions of the wall and fractions are reported in Fig. 7.

\subsection{Heat transfer equation and boundary conditions}

The temperature field in the object obeys the unsteady heat transfer equation given by

$$
\rho C_{p} \frac{\partial T}{\partial t}=\nabla \cdot(k \nabla T)+\dot{q},
$$

in which $T$ is the temperature, $t$ the time, $\rho$ the density, $C_{p}$ the specific heat and $k$ the thermal conductivity. The source term $\dot{q}$ will be detailed below. A perfect contact is assumed between the polymer strands and between the part and the substrate. This is a strong hypothesis as the presence of porosity is practically always seen in FFF. For numerical simplicity the porosity in the part is not taken into account in the meshing. However the thermal conductivity of the polymer is modified to take into account the volumetric porosity. This will be detailed in Subsection 5.1.

As previously explained, the deposition of polymer fractions is performed by successively changing the properties of the domain from air to ABS polymer. Each fraction is deposited at a uniform temperature equal to the extrusion temperature $T_{\text {ext }}$. The substrate is heated at a given temperature equal to $T_{\text {sub }}$. The air surrounding the building object has a constant temperature equal to $T_{\text {air }}$. The surrounding air is not meshed in the domain but taken into account through heat transfer coefficients.

Two boundary conditions are defined to take into account the heat transfer with the surrounding air. Along the surface of the solid domain, denoted as boundary condition 1 in Fig. 8, a Fourier boundary condition given by the following relation

$$
-k \nabla T \cdot n=h_{\text {surf }}\left(T-T_{\text {air }}\right)
$$

is used with $n$ the unit outward normal, $h_{\text {surf }}$ the heat transfer coefficient and $T_{\text {air }}$ the air temperature assumed constant. 


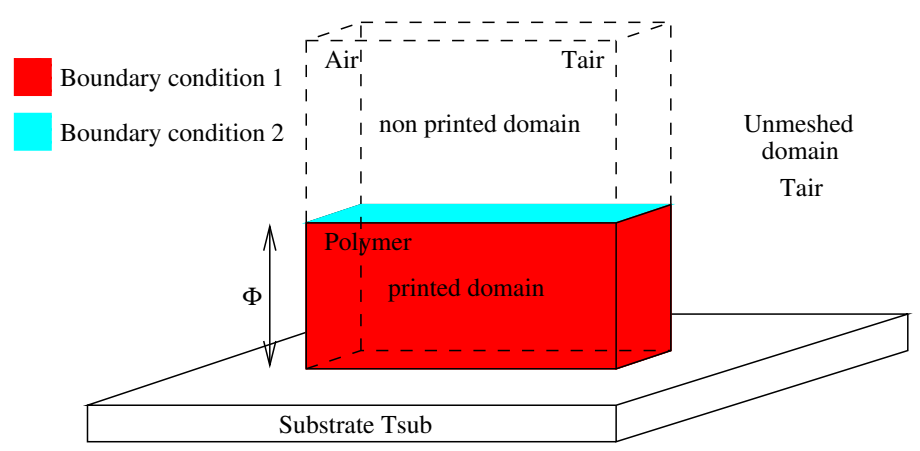

Fig. 8: Sketch of the domain with the two kinds of boundary conditions used in numerical computations.

The convective heat transfer between the top surface of the printing object and the surrounding air has also to be taken into account. This surface is represented as boundary condition 2 in Fig. 8. Since this boundary is immersed in the computational domain, the boundary condition is seen as a heat loss $\dot{q}$ introduced in equation (4). This sink term is applied in the whole layer in printing written as follows

$$
\dot{q}=\frac{h_{\mathrm{top}}\left(T_{\mathrm{air}}-T\right)}{\Delta H},
$$

with $h_{\text {top }}$ the convective heat transfer coefficient with the surrounding air above the printed object.

\section{Experimental results}

4.1 Influence of the printing velocities on strand geometry

The influence of the printing parameters $U$ and $V$ on the strand geometry is investigated. The behaviour of the strand cross-section area with the printing ratio $U / V$ is known by conservation of the volume in the liquefier:

$$
A=\frac{\pi D^{2}}{4} \frac{U}{V}
$$

with $A$ the strand cross-section area. This relation is checked by measuring $A$ for various ratio $U / V$ at different nozzle velocity $V$. Knowing the polymer density, the average strand cross-section area is calculated by weighting single strands of known length. As shown in Fig. 9, equation (7) is valid in the experimental field of the study.

An approximation of the strand geometry is searched to estimate the strand's height and width depending on printing velocities. The strand's width $W$ and height $H$ is first measured using optical microscopy for different ratio $U / V$ and different nozzle velocity $V$. The measured dimensions are then used to calculate the strand cross-section areas assuming different geometrical shapes. The behaviour of the cross-section area with $U / V$ is then compared to equation (7). This enables to know which geometrical shape gives the best approximation of the actual strand morphology. According to Comminal et al. [5], the chosen geometrical 


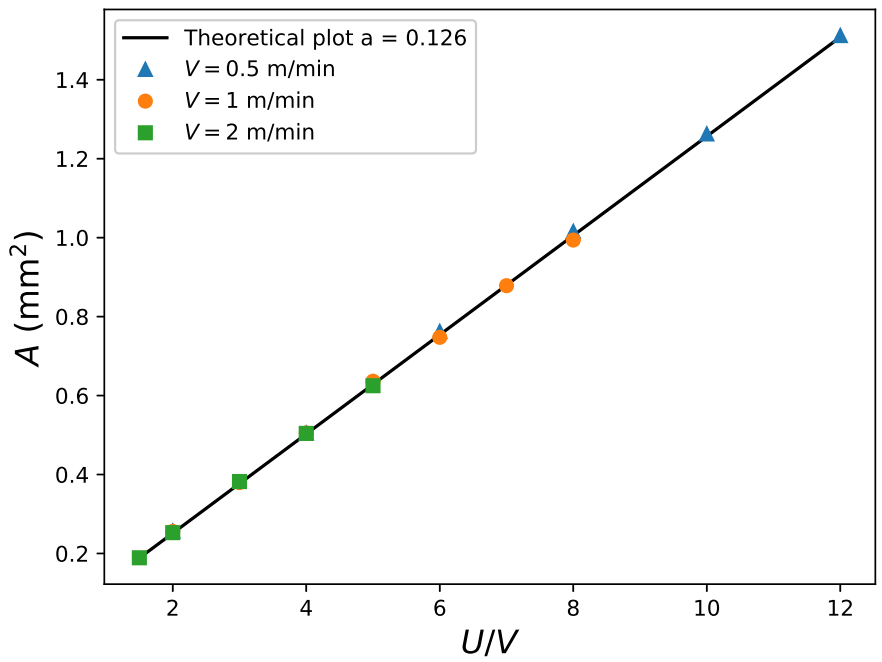

Fig. 9: $A$ as a function of the printing parameters $U / V$. Comparison with the theoretical plot described in equation (7).

\begin{tabular}{cc}
\hline Geometrical shape & Area \\
\hline Rectangle & $A=W H$ \\
Ellipse & $A=\frac{\pi}{4} W H$ \\
Oblong & $A=W H-H^{2}\left(1-\frac{\pi}{4}\right)$ \\
\hline
\end{tabular}

Table 2: Cross-section area of the geometrical shapes chosen to approximate the geometry of the strand with $W$ the width and $H$ the height of the strand.

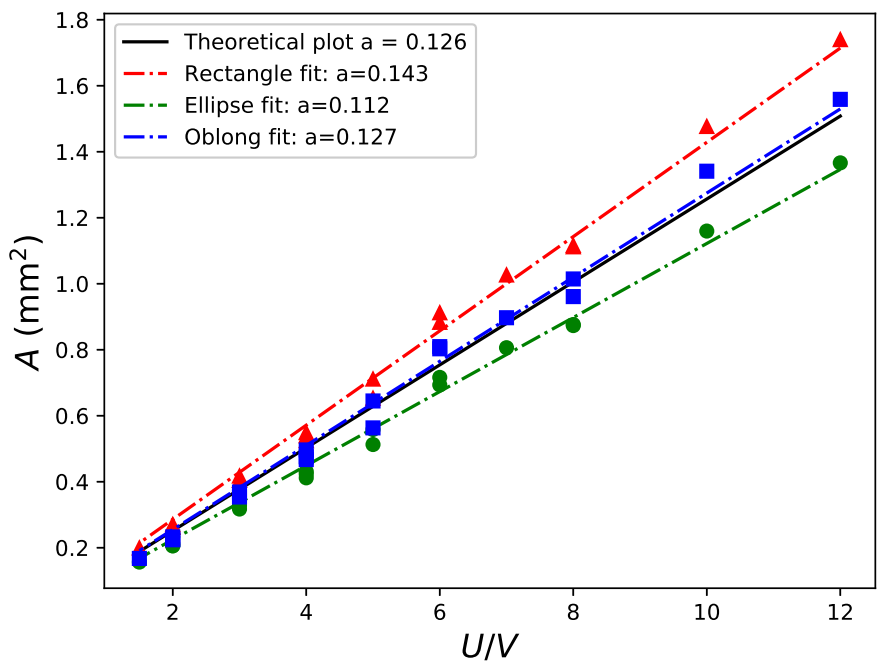

Fig. 10: Cross-section area $A$ as a function of $U / V$ assuming three different geometrical shapes. Comparison with the solution given by (7) with $a$ the slope of the linear function. 


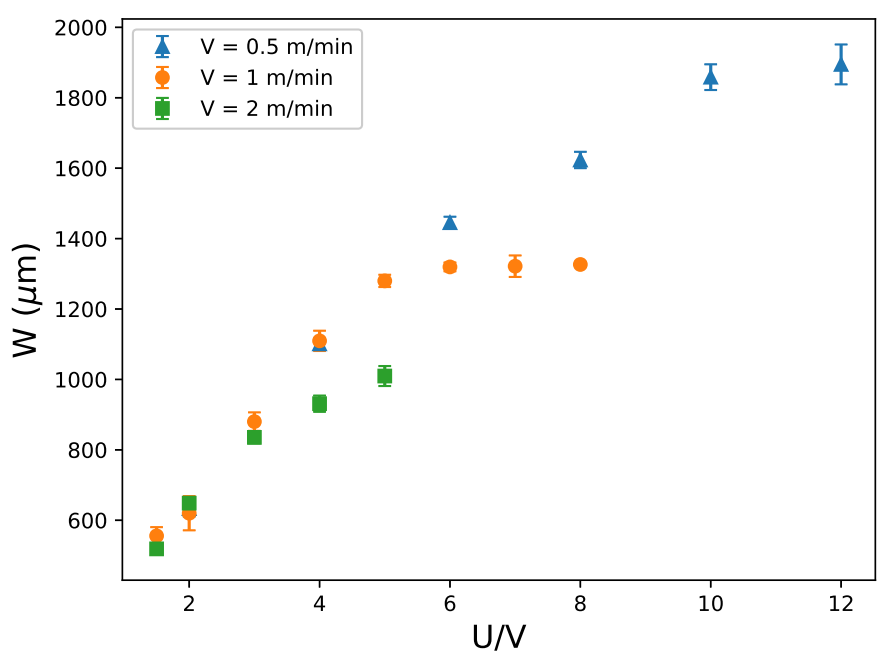

Fig. 11: $W$ as a function of $U / V$ for three values of the printing velocity $V$ equal to $0.5,1$ and $2 \mathrm{mmin}^{-1}$.

shapes are the rectangle, the ellipse and the oblong. The area formula of each cross-section are recalled in Table 2.

Fig. 10 depicts the area of the cross-section using the three shapes. The oblong shape predicts accurately the strand cross-section area as a function of the printing ratio $U / V$. Indeed, the difference of the slope between equation (7) and the oblong area is only $0.8 \%$. The oblong geometry can thus be used to predict the strand geometry based on printing velocities $U$ and $V$ with minimum error of the actual strand area. The following relation can thus be used to predict the strand's height and width based on the printing velocities:

$$
W H-H^{2}\left(1-\frac{\pi}{4}\right)=\frac{\pi D^{2}}{4} \frac{U}{V}
$$

The strand's width is plotted as a function of $U / V$ for three printing velocities $V$ in Fig. 11. The strand's width depends significantly on the printing velocity $V$. At small $U / V$, $W$ presents the same trend whatever the printing velocity. When $U / V$ increases, $W$ reaches a threshold depending on the printing velocity. This threshold is observed when the extrusion velocity $U$ is higher than $5 \mathrm{mmin}^{-1}$ for the three printing velocities. This is a consequence of the insufficient melting of the polymer in the liquefier. Indeed, with the increase of the extrusion velocity, the polymer is lesser and lesser heated in the liquefier as it has been pinpointed in $[12,16]$. This would lead to higher viscosity in the centre of the strand, limiting the spread of deposition.

The behaviour of $W$ with printing ratio $U / V$ is thus considered for $U \leq 5 \mathrm{mmin}^{-1}$ in Fig. 12. Hebda et al. [9] have presented a master curve to estimate the strand's width based on printing parameters $U$ and $V$. Based on their study, the master curve is modified to fit our measurements defined as follows

$$
W=\alpha D \sqrt{\frac{U}{V}}+C
$$




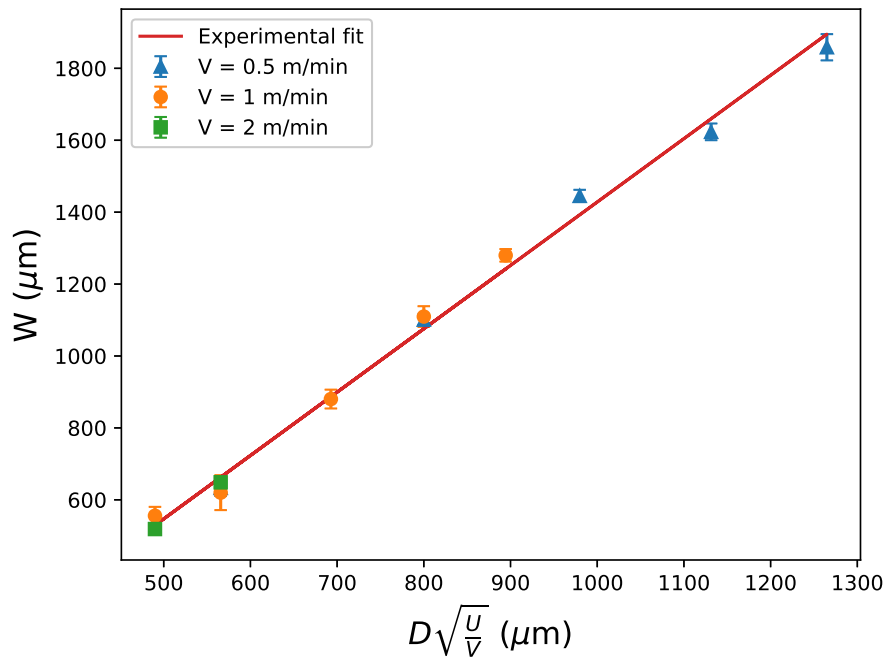

Fig. 12: $W$ as a function of $U / V$ for $U \leq 5 \mathrm{~m} \mathrm{~min}^{-1}$ and experimental fit according to Hebda et al. [9].

with $\alpha$ a pre-factor and $C$ a constant determined by linear regression based on our experimental data. These parameters depend on the polymer and the printer. The fitted parameters are $\alpha=1.76$ and $C=-334.2$ for the ABS polymer. The experimental fit is presented in Fig. 12.

The strand's height measured experimentally for the three printing velocities is plotted in Fig. 13 as a function of $U / V$. Whatever $V, H$ presents a same behaviour as a function of $U / V$. Using the two equations (9) in (8) it is possible to estimate $H$. Indeed, the substitution of (9) in (8) gives the following quadratic equation

$$
-H^{2}\left(1-\frac{\pi}{4}\right)+H\left(\alpha D \sqrt{\frac{U}{V}}+C\right)-\frac{\pi D^{2}}{4} \frac{U}{V}=0 .
$$

By solving the previous equation, $H$ is determined as a function of $U / V$. The prediction using our fitted parameters is provided in Fig. 13 in solid line. The relation given by (10) can provide a first approximation of the strand's height when printing a piece. The use of equations (8) and (9) enables to better calibrate the deposition path of a printed object without performing actual measurements of the strand.

4.2 Influence of the printing parameters on cooling kinetics

The influence of the nozzle velocity $V$ is studied by measuring the inter-layer temperature for three nozzle velocities. The extrusion velocity $U$ is equal to $4 \mathrm{~m} / \mathrm{min}$. Fig. 14 shows the temperature behaviour for the three nozzle velocities $V$ equal to $0.5,1$ and $2 \mathrm{~m} \mathrm{~min}^{-1}$. Temperature profiles are plotted as a function of time divided by the period of layer deposition $\tau_{\text {dep. }}$. This period is equal to 48,24 and $12 \mathrm{~s}$ for $V$ equal to $0.5,1$ and $2 \mathrm{mmin}^{-1}$ respectively. 


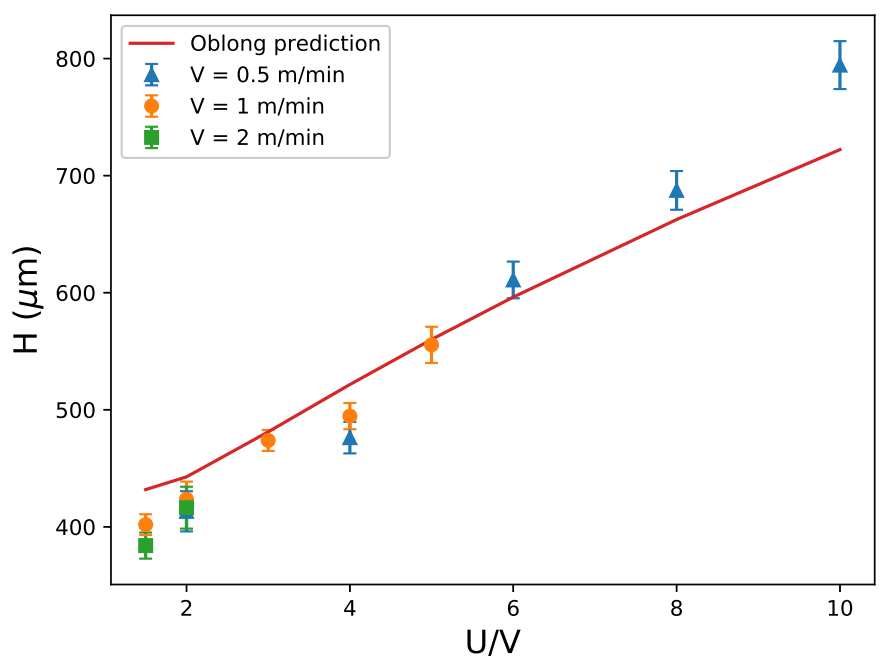

Fig. 13: $H$ as a function of $U / V$ for $U \leq 5 \mathrm{mmin}^{-1}$. Comparison with $H$ calculated using equations (9) and (7) for different strand geometry. The fitting parameters are $\alpha=1.76$ and $C=-334.2$ for the studied ABS polymer.

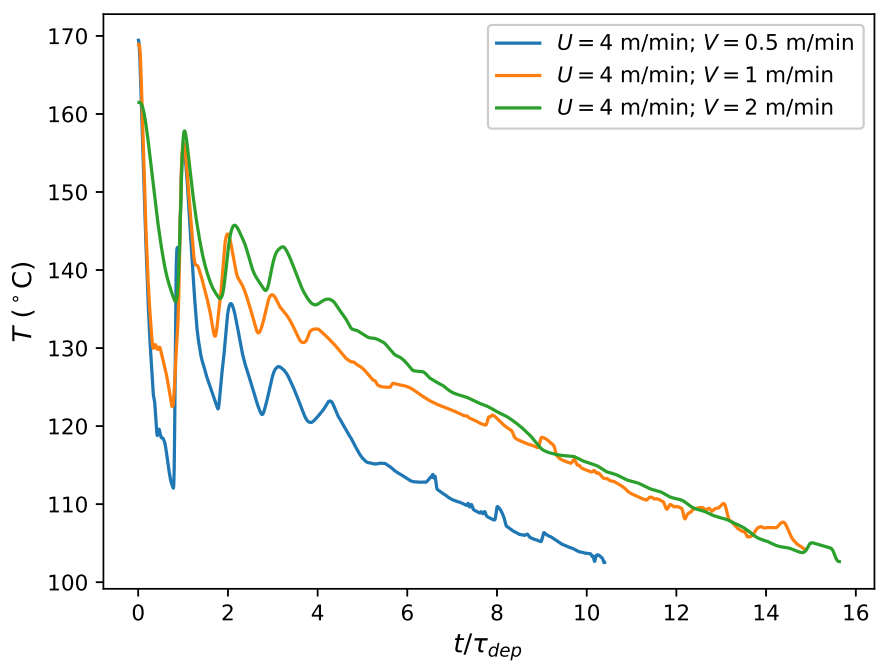

Fig. 14: Temperature recorded by the thermocouple as a function of $t / \tau_{\text {dep }}$ for an extrusion velocity of $4 \mathrm{~m} \mathrm{~min}^{-1}$ and for three nozzle velocities $V$ equal to $0.5,1$ and $2 \mathrm{~m} \mathrm{~min}^{-1}$. 


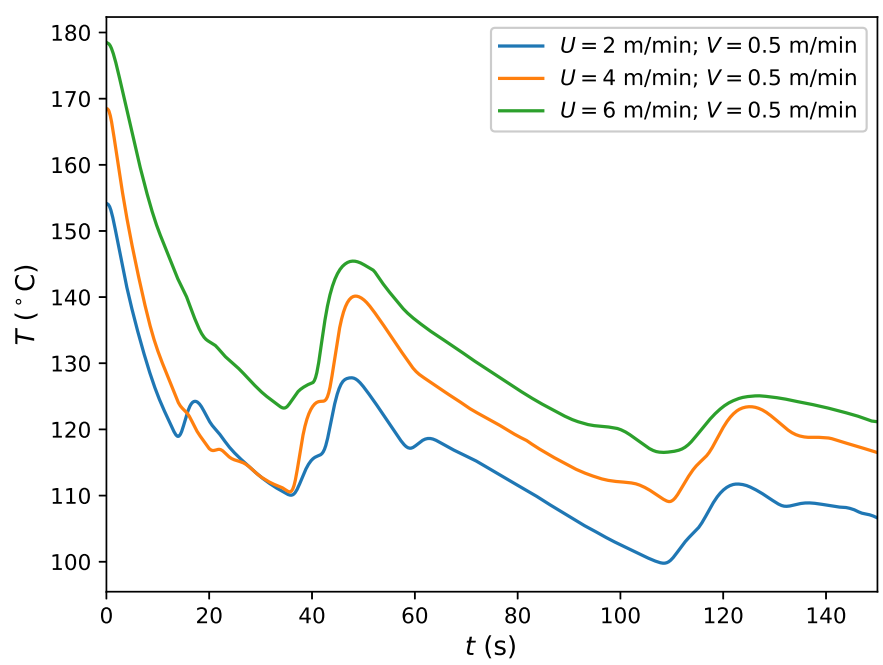

Fig. 15: Temperature recorded by the thermocouple as a function of time for a nozzle velocity of $0.5 \mathrm{~m} \mathrm{~min}^{-1}$ and for three extrusion velocities $U$ equal to 2,4 and $6 \mathrm{~m} \mathrm{~min}^{-1}$.

The temporal origin in Fig. 14 is chosen when the polymer filament is in contact of the thermocouple. The highest temperature is thus reached at $t=0$. The first decrease corresponds to the cooling of the polymer just after its deposition on the thermocouple. It is represented by strand $(p, 2)$ in Fig. 4 . The other peaks correspond to the deposition of new layers with a period equal to $\tau_{\text {dep }}$. It is represented by strand $(p+1 ; 2),(p+2 ; 2)$, etc in Fig. 4. This trend shows that a strand is more influenced by vertical deposition than horizontal deposition. The heating and cooling magnitudes are decreasing as deposition occurs farther from the thermocouple.

According to our inter-layer measurements, the printing of a layer $p$ has a major impact on the layers $(p-1)$ and below. Seppala and Migler [17] have measured the temperature of each layer by Infrared thermography. According to their study, layers below $(p-1)$ are not sufficiently heated to stay above $T_{g}$ over an extended period of time. However, Fig. 14 shows that the inter-layer temperature stays above $T_{g}$ during the deposition of multiple layers. The time required to observe an inter-layer temperature below $T_{g}$ is around 120-265 s. Over this period, adhesion between strands is possible at a such temperature leading to a good welding.

Fig. 15 shows the temperature behaviour for $U$ equal to 2,4 and $6 \mathrm{mmin}^{-1}$ at a constant nozzle velocity $V$ equal to $0.5 \mathrm{~m} \mathrm{~min}^{-1}$. The temporal origin is also defined when the polymer filament is in contact with the thermocouple. Only the first two periods are recorded for this series of measurements. For $U=2 \mathrm{~m} \mathrm{~min}^{-1}$, a small increase in temperature is noticed when $t \sim 20 \mathrm{~s}$. At this velocity, the strand size is the smallest due to the low flow rate. Therefore, the thermocouple is more sensitive to the increase of temperature due to the deposition of polymer near its location. A similar event is also seen during the second period of deposition at $t \sim 60 \mathrm{~s}$.

The temperature recorded by the thermocouple increases with the extrusion velocity. This feature seems counter-intuitive since the residence time of the polymer in the extruder decreases with $U$. Consequently, the heating of the polymer should decrease with $U$. Ac- 
(a)

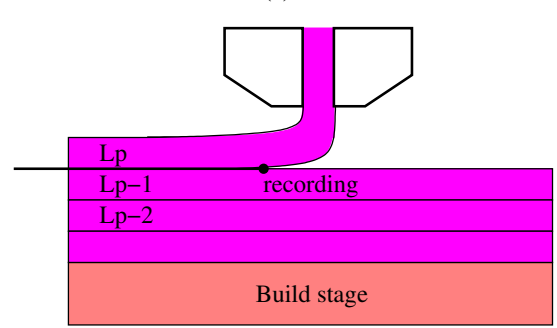

(b)

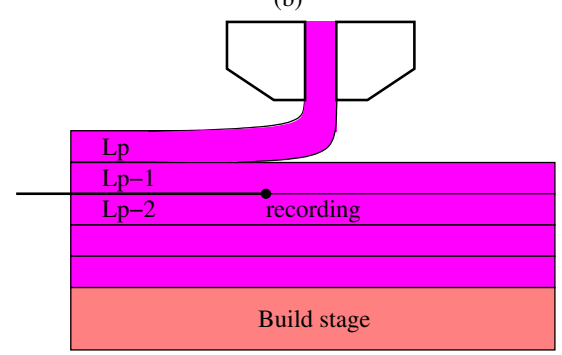

Fig. 16: Measurement areas between layers $L_{p}$ and $L_{p-1}$ (a) and between layers $L_{p-1}$ and $L_{p-2}(\mathrm{~b})$.

cording to Peng et al. [15], the increase of the extrusion velocity from 2 to $6 \mathrm{~m} \mathrm{~min}^{-1}$ leads to the decrease of the exit temperature. Pigeonneau et al. [16] performed thermal computations of polymer flowing through the liquefier. For high extrusion velocities, the temperature is also lower at the centre of the liquefier. However, thermal heterogeneity stays moderate and the average temperature at the exit of the liquefier is close to the preset extrusion temperature. The variation of temperature observed in Fig. 15 is explained by the amount of deposited polymer. As already mentioned, the amount of extruded material is proportional to $U$. Moreover, the stored energy is linked to the amount of deposited polymer. Therefore, the temperature increases with the amount of extruded material.

The same feature is seen in Fig. 14. Indeed, maximum temperature is observed for the lowest nozzle velocity corresponding to the largest filament size. Thomas and Rodríguez [21] also concluded that the strand cross-section area has a major impact on the cooling kinetics. Moreover, the thermocouple is better covered by the polymer when the filament size is larger. However, the extended deposition time for low nozzle velocity leads to the lowest temperature after complete deposition of the layer. This is due to continuous energy loss along the exposed surfaces, which corresponds to the case $V=0.5 \mathrm{~m} \mathrm{~min}^{-1}$ in Fig. 14.

\subsection{Influence of strand size on cooling kinetics}

In the following subsection, the influence of the strand size on cooling kinetics is quantified by varying the extrusion velocity $U$. To obtain a sufficiently wide filament, a constant nozzle velocity $V$ is set equal to $0.5 \mathrm{~m} \mathrm{~min}^{-1}$. The experimental set-up is slightly modified to study the cooling of inter-layer denoted $L_{p} / L_{p-1}$ and $L_{p-1} / L_{p-2}$ in Fig. 16.

Thermocouple measurements of inter-layer $L_{p} / L_{p-1}$ and $L_{p-1} / L_{p-2}$ for different extrusion velocities have been carried out. After the printing of the layer $L_{p}$ and the covering of the thermocouple, a standby of eight seconds is operated. The cooling kinetics of a layer is then determined. Fig. 17 presents an example of the temperature behaviour in which the successive heating and cooling are seen. The inter-layer cooling temperature $L_{p} / L_{p-1}$ is then fitted by an exponential time decay written as follows

$$
T(t)=T_{\text {air }}+\left(T_{0}-T_{\text {air }}\right) \exp \left(-\frac{t}{\tau}\right),
$$

with $T$ the temperature, $T_{\text {air }}$ the surrounding air temperature, $T_{0}$ the initial temperature, $t$ the time and $\tau$ the characteristic time of cooling. The comparison between experimental 


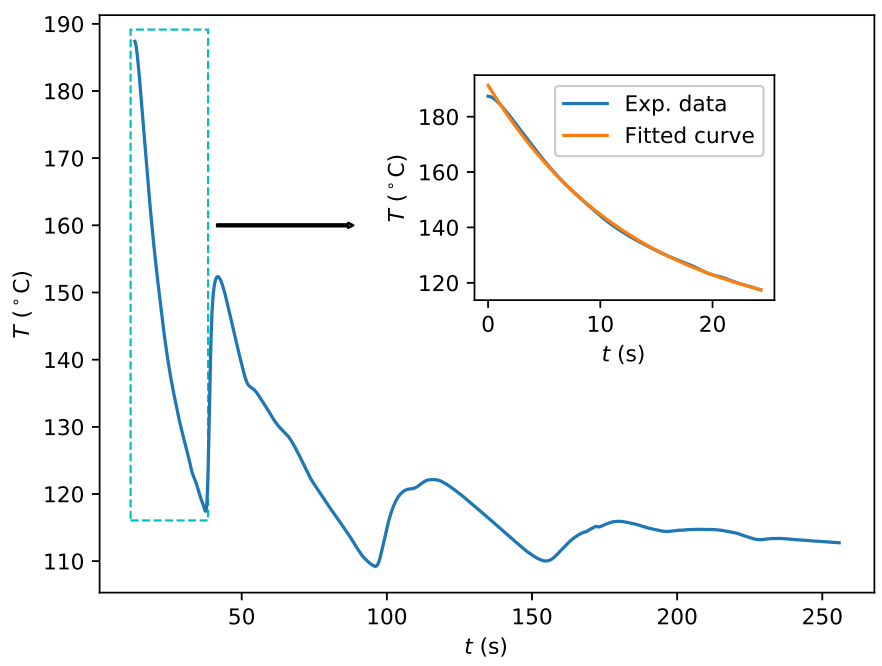

Fig. 17: Example of the temperature behaviour of a printed strand. The insert depicts the comparison between the experimental result (blue) and the relation (11) of the first cooling (orange).

data and the fitting curve is shown in the insert of Fig. 17. The same procedure is repeated between the layers $L_{p-1}$ and $L_{p-2}$ corresponding to the situation shown in Fig. 16(b).

Fig. 18 shows the behaviour of the characteristic time $\tau$ of equation (11) from the cooling of inter-layer $L_{p} / L_{p-1}$ and $L_{p-1} / L_{p-2}$, referred as first and second peaks respectively. Different printing velocities are investigated to vary the strand size. The cooling kinetics is thus plotted as a function of the strand cross-section area. Measurements of the width and height of the printed strand cross-section are done using an optical microscope as explained in subsection 2.3. The cross section of the strand is assumed as an oblong to estimate its area. Multiple tests are repeated and the average characteristic time is calculated for the different printing conditions.

As expected, Fig. 18 shows that $\tau$ is smaller for the cooling of inter-layer $L_{p} / L_{p-1}$ than for the cooling of inter-layer $L_{p-1} / L_{p-2}$. For a given strand size, the difference in the cooling time $\tau$ is explained by the nature of the heat transfer. The cooling of inter-layer $L_{p} / L_{p-1}$ is mainly driven by the convective heat transfer from the upper boundary with the surrounding air. Conversely, the cooling of inter-layer $L_{p-1} / L_{p-2}$ is mainly driven by the heat transfer with lateral boundaries. This shows that different heat transfer phenomena are seen depending on the step of the process. The development of an analytic model is thus complicated for this process. Therefore, numerical simulations are achieved to predict thermal history of FFF printed parts.

\section{Numerical computation and comparison with experimental results}

In the following section, comparisons between experimental measurements and numerical simulations are achieved to assess the numerical model. A first case is studied to determine 


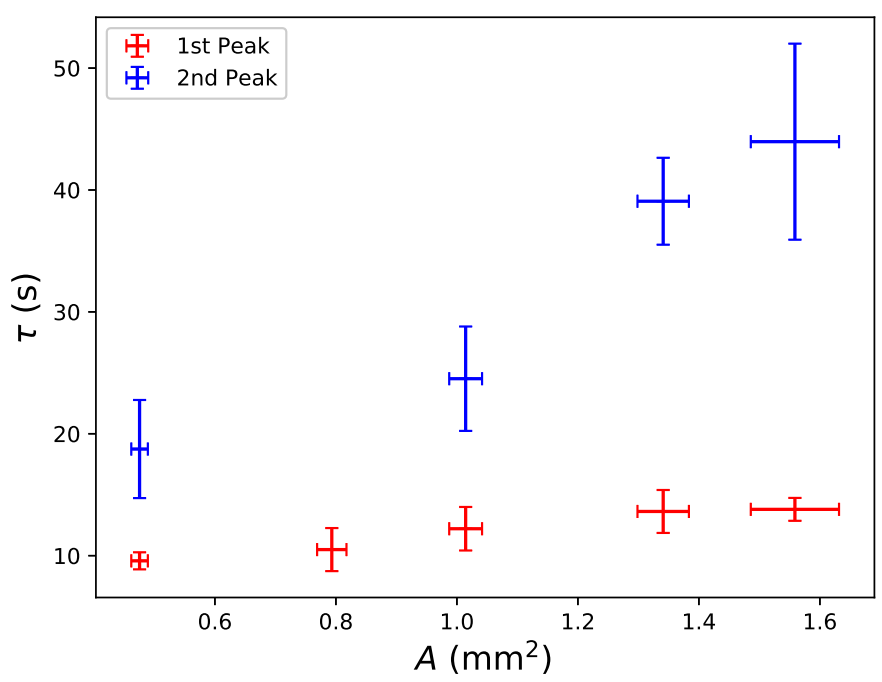

Fig. 18: Cooling time $\tau$ of equation (11) as a function of the strand cross-section area $A$ for the inter-layer $L_{p} / L_{p-1}$ (first peak) and $L_{p-1} / L_{p-2}$ (second peak), for $V=0.5 \mathrm{~m} / \mathrm{min}$.

the numerical parameters minimising the errors with experimental results. The same set of parameters is then used on different cases to assess the validity of the model.

\subsection{Numerical computations}

Numerical computations are achieved for three cases characterised by the printing parameters provided in Table 3. As mentioned in section 2.3, the strand dimensions depend on the velocities $U$ and $V$. Subsection 4.3 have shown that the strand size is a predominant parameter of the cooling kinetics. Computations are thus done for a smaller and a larger strand size to validate the thermal parameters found in case 1 . Special attention is taken to ensure mass conservation. The strand is assumed rectangular in the numerical computations. Therefore the mass balance between the flow in the extruder and the motion of the strand gives

$$
\Delta H=\frac{\pi D^{2}}{4 \Delta W} \frac{U}{V}
$$

with $D$ the extruder diameter at the exit, see Fig. 1. Completed by optical microscopy measurements, $\Delta H$ and $\Delta W$ are also provided in Table 3.

The strand morphology is assumed rectangular for all numerical computation. However subsection 4.1 have shown that the oblong geometry better describes the geometry of the strand. The volume loss when considering an oblong compared to a rectangle is assumed as porosity. Therefore the overall thermal conductivity of the printed part is modified to take into account porosity in the medium. According to Bejan [2], the thermal conductivity of the medium can be calculated as followed:

$$
k_{m}=\left(1-\varphi_{p}\right) k_{s}+\varphi_{p} k_{f}
$$




\begin{tabular}{ccccc}
\hline Printing parameters & Unit & Case 1 & Case 2 & Case 3 \\
\hline$U$ & $\mathrm{mmin}^{-1}$ & 4.82 & 5.36 & 4.3 \\
$V$ & $\mathrm{mmin}^{-1}$ & 1.2 & 0.663 & 2.14 \\
$\Delta H$ & $\mathrm{~mm}$ & 0.5 & 0.67 & 0.5 \\
$\Delta W$ & $\mathrm{~mm}$ & 1 & 1.5 & 0.5 \\
$W$ & $\mathrm{~mm}$ & 4 & 6 & 2 \\
$L$ & $\mathrm{~mm}$ & 100 & 100 & 100 \\
$t_{l}$ & $\mathrm{~s}$ & 5 & 9 & 2.8 \\
$t_{f}$ & $\mathrm{~s}$ & 1.25 & 2.25 & 0.7 \\
$\varphi_{p}$ & $\%$ & 10.7 & 9.6 & 21.5 \\
$k_{m}$ & $\mathrm{~W} \mathrm{~m}^{-1} \mathrm{~K}^{-1}$ & 0.164 & 0.165 & 0.147 \\
\hline
\end{tabular}

Table 3: Computation parameters and corresponding times for the three printing conditions.

with $k_{m}$ the thermal conductivity of the medium, $k_{s}$ the thermal conductivity of the solid, in our case the ABS and $k_{f}$ the thermal conductivity of air and $\varphi_{p}$ the volumetric porosity of gas phase. The thermal conductivity of the medium as well as the volumetric porosity is provided in Table 3 for all cases.

The walls are printed with our $3 \mathrm{~d}$ printer in the working conditions provided in Table 3. The thermal measurements are based on the same experimental procedure as discussed in subsection 2.4. The printing of the wall continues until the inter-layer temperature reaches $T_{g}$. Therefore, the final height depends on the printing conditions. Using the Cartesian coordinates framework represented in Fig. 7a, the locations of the thermocouple are $x=15$, $y=-0.5, z=5 \mathrm{~mm}$ for case $1, x=15, y=-0.75, z=4.7 \mathrm{~mm}$ for case 2 and $x=15$, $y=-0.25, z=5 \mathrm{~mm}$ for case 3 . The printed wall height does not exceed $3 \mathrm{~cm}$.

The temperature of the substrate is set at $T_{\text {sub }}=94{ }^{\circ} \mathrm{C}$. Following measurements in the surrounding air, $T_{\text {air }}$ is set equal to $50{ }^{\circ} \mathrm{C}$. The temperature of the polymer fractions is set equal to $T_{\mathrm{ext}}=200^{\circ} \mathrm{C}$ in the numerical computations. Finally, the only two parameters controlling the temperature behaviour are the heat transfer coefficients $h_{\text {surf }}$ and $h_{\text {top }}$.

\subsection{Comparison with experimental results}

Fig. 19 depicts the temperature as a function of time localised on the thermocouple position for case 1 . The blue solid line corresponds to the numerical results while the orange line is the temperature recorded experimentally. To help the reading of Fig. 19, the glass transition temperature (green solid line) is also drawn.

The successive heating and cooling simulated are in good agreement with experimental measurements. Nevertheless, the numerical results seem more sensitive to adjacent depositions of new hot filaments. Indeed, heating is numerically observed when deposition of the adjacent strand occurs. The inter-layer reheating due to the deposition of a new layer is strongly reduced once it is covered by more than three layers. After an extended period of time, the steady-state cooling observed experimentally is numerically very well reproduced.

To observe a good agreement with experimental results in case $1, h_{\text {surf }}$ and $h_{\text {top }}$ are set equal to 13 and $8 \mathrm{Wm}^{-2} \mathrm{~K}^{-1}$ respectively. These values can be compared with the data given in the heat transfer literature. For natural convection of external flows over a horizontal surface, Jaluria [10] provides an empirical correlation depending on the Rayleigh number based on air properties. If the characteristic length is taken equal to the width of the wall and by taking the properties of air at $T_{\text {air }}=50^{\circ} \mathrm{C}$, the heat transfer coefficient is approximately equal to $34 \mathrm{Wm}^{-2} \mathrm{~K}^{-1}$. Bellehumeur et al. [3] studied the influence of the convective heat 


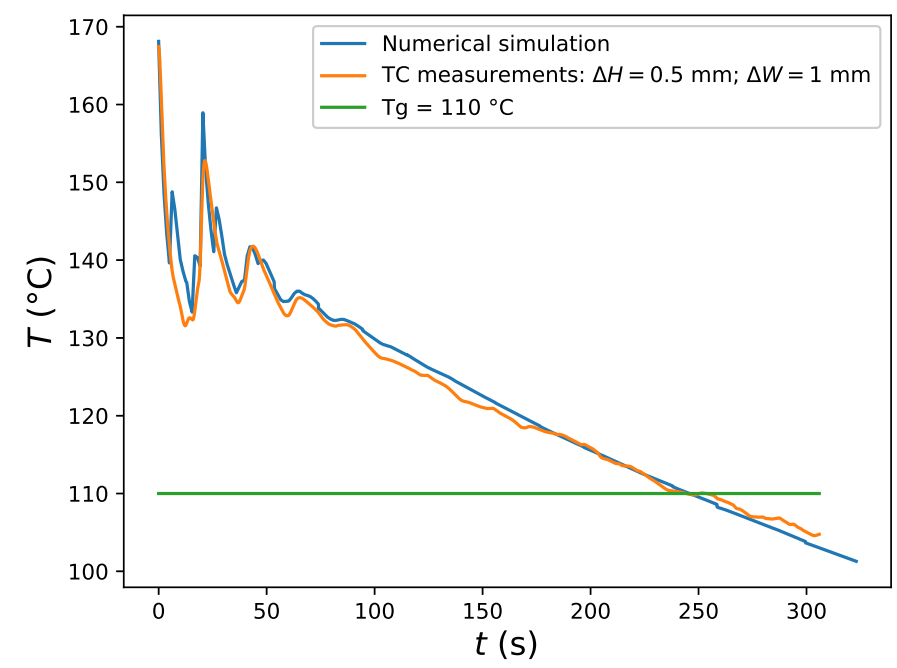

Fig. 19: Inter-layer temperature as a function of time for case 1. Comparison between numerical and experimental results. $U=4.82 \mathrm{~m} / \mathrm{min}$ and $V=1.2 \mathrm{~m} / \mathrm{min}$

transfer coefficient on the strand temperature. For $T_{\text {air }}=70^{\circ} \mathrm{C}$, the heat transfer coefficient is taken equal to $30 \mathrm{Wm}^{-2} \mathrm{~K}^{-1}$ to compute the temperature profile of a single deposited strand. To match with their experimental measurements, Costa et al. [7] set a convective heat transfer coefficient at $62 \mathrm{Wm}^{-2} \mathrm{~K}^{-1}$. Consequently, the heat transfer coefficients found in our work are relatively smaller than values used in these previous contributions.

Fig. 20 gives the inter-layer temperature as a function of time for case 2 and 3 . The numerical results have been obtained with the same heat transfer coefficients as case 1 . The maximum temperature of each peak matches very well with the experimental measurements. As previously observed, the strands obtained numerically are more sensitive to the deposition of adjacent strands. The strand size of case 3 is smaller than case 1 explaining this increase of sensitivity. The strand size of case 2 is larger than case 1 and 3 explaining lower sensitivity.

The time for which the polymer temperature reaches $T_{g}$ is one of the major criteria to characterise the inter-layer adhesion. According to McIlroy and Olmsted [14], the interdiffusion between strands occurs until the welding temperature is lower than $T_{g}$. Below $T_{g}$, the molecular motions are too restrained to enable diffusion at the interface. For all tested cases, the time for which the polymer reaches $T_{g}$ is very well reproduced with numerical simulation. A cooling rate is calculated to compare numerical computations with measurements. The cooling rate is defined as follows

$$
\dot{T}_{\text {ref }}=\frac{\Delta T}{\Delta t}=\frac{\left|T_{g}-T_{0}\right|}{t_{T_{g}}-t_{0}}
$$

with $T_{0}$ the inter-layer temperature when the polymer is deposited at $t_{0}$. The time for which the inter-layer temperature reaches $T_{g}$ is defined by $t_{T_{g}}$. The experimental and numerical cooling rates defined by (14) are summarised in Table 4 . A good agreement is found between the experimental and numerical results. This criterion does not give information about how 
(a) Case 2: $U=5.36 \mathrm{mmin}^{-1}, V=0.663 \mathrm{mmin}^{-1}$

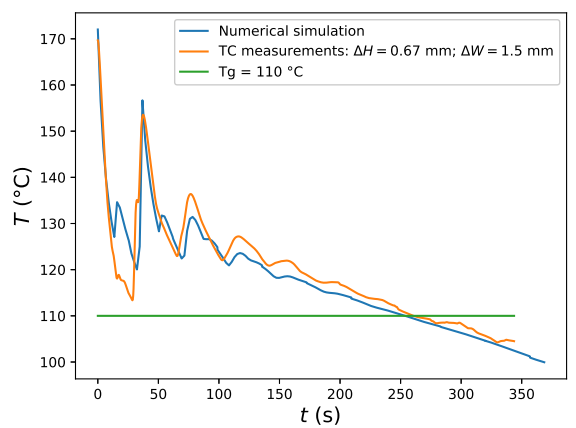

(b) Case 3: $U=4.3 \mathrm{mmin}^{-1}$ and $V=2.14 \mathrm{mmin}^{-1}$

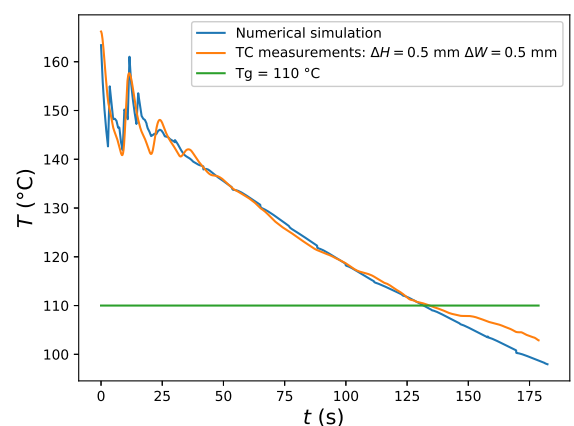

Fig. 20: Inter-layer temperature as a function of time for cases 2 and 3. Comparison between numerical and experimental results.

\begin{tabular}{ccc}
\hline Case & Experimental cooling rate $\left(\mathrm{K} \mathrm{min}^{-1}\right)$ & Numerical cooling rate $\left(\mathrm{K} \mathrm{min}^{-1}\right)$ \\
\hline Case 1 & $14.1 \pm 1.1$ & 14.2 \\
Case 2 & $13.8 \pm 1.1$ & 14.6 \\
Case 3 & $25.1 \pm 1.5$ & 24.3 \\
\hline
\end{tabular}

Table 4: Comparison of the experimental and numerical cooling rate computed for all studied cases.

the temperature behaves during the cooling. However, it shows that the overall cooling rate is very well described by the numerical simulation.

For the three cases, the maximum temperatures seen during the two first deposited layers match well with the thermocouple measurements. Good predictions of the maximum temperature is very important to predict the adhesion between the strands. Ko et al. [11] have given parameters to calculate the degree of adhesion for a PC-ABS co-polymer used in FFF. These parameters are based on a relation developed by Yang and Pitchumani [23]. The given parameters show that most of adhesion occurs when maximum temperature is reached, i.e. when the layer is deposited and when it is covered by a new layer. The degree of adhesion can thus be calculated with our model as the maximum temperatures are well described.

\section{Conclusion}

This work has been devoted to the cooling kinetics of an object printed by the Fused Filament Fabrication process. Simple walls are printed in ABS polymer. Thermocouples are introduced in the wall to record the temperature between two layers of polymer. A dedicated numerical tool is also used to investigate thermal behaviour during the printing process.

Measurements of the strand shape have been done for various printing velocities. A relation predicting the strand size based on printing parameters have been described based on these measurements. Thermal measurements have been achieved to study the influence of strand shape on cooling kinetics. The inter-layer temperature undergoes a cycle of heating and cooling due to the deposition of new layers of melted polymer. When the number of layers becomes higher than three, the inter-layer temperature decreases slowly due to the 
heat transfer with the surrounding air. It is noteworthy that the strand geometry controls the cooling kinetics.

The numerical model predicts well the overall temperature behaviour of a printed object. Moreover, it enables to obtain representative information of temperature over an extended period of time. The numerical tool has been assessed by experimental measurements for different printing velocities, inducing different strand sizes. The model also takes into consideration the porosity induced by the process. To the author knowledge, it is the first time that experimental measurements of the temperature in a representative object printed with the FFF process have been successfully compared to numerical computations. The model can be used to predict the bonding quality between adjacent strands. The relation predicting strand size based on printing velocities can be coupled with the numerical model to predict the thermal behaviour of the process. This kind of numerical computations could be a useful tool to limit the number of experiments. Moreover, the use of this tool is also expected to enable the optimisation of object shapes.

Improvements of the numerical tool are still required to better reproduce the first cooling of a freshly deposited strand. Indeed, the heat sink is described by a volumetric source term while in reality, it is a surface sink. Numerical tool can be extended in two main axis. First, the degree of healing is an important criterion to evaluate the welding between the printed filaments of polymer. Based on the thermal history, this criterion can be easily computed with the numerical tool. The second direction is to predict the mechanical behaviour to have an estimation of the residual stresses and of the warpage.

Acknowledgements The authors are indebted to J.-F. Agassant for his advice during the preparation of this article and the various discussions about the $3 \mathrm{~d}$-printing process.

\section{Conflict of interest}

The authors declare that they have no conflict of interest.

\section{References}

1. Agassant, J.F., Pigeonneau, F., Sardo, L., Vincent, M.: Flow analysis of the polymer spreading during extrusion additive manufacturing. Addit. Manuf. 29, 100794 (2019). DOI https://doi.org/ 10.1016/j.addma.2019.100794. URL http://www.sciencedirect.com/science/article/pii/ S2214860419304932

2. Bejan, A.: Porous media. In: Heat transfer handbook, chap. 15, pp. 1131-1180. John Wiley \& Sons, Inc. (2003)

3. Bellehumeur, C., Li, L., Sun, Q., Gu, P.: Modeling of bond formation between polymer filaments in the fused deposition modeling process. J. Manuf. Processes 6(2), 170-178 (2004). DOI 10.1016/ s1526-6125(04)70071-7. URL http://dx.doi.org/10.1016/S1526-6125(04)70071-7

4. Bikas, H., Stavropoulos, P., Chryssolouris, G.: Additive manufacturing methods and modelling approaches: a critical review. Int. J. Adv. Manuf. Technol. 83(1-4), 389-405 (2015). DOI 10.1007/ s00170-015-7576-2. URL http://dx.doi.org/10.1007/s00170-015-7576-2

5. Comminal, R., Serdeczny, M.P., Pedersen, D.B., Spangenberg, J.: Numerical modeling of the strand deposition flow in extrusion-based additive manufacturing. Addit. Manuf. 20, 68 - 76 (2018). DOI https://doi.org/10.1016/j.addma.2017.12.013. URL http://www.sciencedirect.com/science/ article/pii/S2214860417305079

6. Costa, S.F., Duarte, F.M., Covas, J.: Thermal conditions affecting heat transfer in FDM/FFE: a contribution towards the numerical modelling of the process. Virtual Phys. Prototy. 10(1), 35-46 (2015). DOI 10.1080/17452759.2014.984042. URL https://doi.org/10.1080/17452759.2014.984042 
7. Costa, S.F., Duarte, F.M., Covas, J.A.: Estimation of filament temperature and adhesion development in fused deposition techniques. J. Mater. Process. Technol. 245, 167-179 (2017)

8. Goh, G.D., Yap, Y.L., Tan, H.K.J., Sing, S.L., Goh, G.L., Yeong, W.Y.: Process-structure-properties in polymer additive manufacturing via material extrusion: A review. Crit. Rev. Solid State Mater. Sci. 45(2), 113-133 (2020). DOI 10.1080/10408436.2018.1549977. URL https://doi.org/10.1080/ 10408436.2018 .1549977

9. Hebda, M., McIlroy, C., Whiteside, B., Caton-Rose, F., Coates, P.: A method for predicting geometric characteristics of polymer deposition during fused-filament-fabrication. Addit. Manuf. 27, 99 - 108 (2019). DOI https://doi.org/10.1016/j.addma.2019.02.013. URL http://www . sciencedirect.com/ science/article/pii/S2214860418308091

10. Jaluria, Y.: Natural convection. In: A. Bejan, A.D. Kraus (eds.) Heat transfer handbook, chap. 7, pp. 525-571. John Wiley \& Sons, Inc. (2003)

11. Ko, Y.S., Herrmann, D., Tolar, O., Elspass, W.J., Brändli, C.: Improving the filament weld-strength of fused filament fabrication products through improved interdiffusion. Addit. Manuf. 29, 100815 (2019)

12. Mackay, M.E., Swain, Z.R., Banbury, C.R., Phan, D.D., Edwards, D.A.: The performance of the hot end in a plasticating 3D printer. J. Rheol. 61(2), 229-236 (2017)

13. McIlroy, C., Graham, R.S.: Modelling flow-enhanced crystallisation during fused filament fabrication of semi-crystalline polymer melts. Addit. Manuf. 24, 323 - 340 (2018). DOI https://doi.org/ 10.1016/j.addma.2018.10.018. URL http://www.sciencedirect.com/science/article/pii/ S2214860418305554

14. McIlroy, C., Olmsted, P.: Disentanglement effects on welding behaviour of polymer melts during the fused-filament-fabrication method for additive manufacturing. Polymer 123, 376 - 391 (2017). DOI https://doi.org/10.1016/j.polymer.2017.06.051. URL http://www.sciencedirect.com/science/ article/pii/S0032386117306213

15. Peng, F., Vogt, B.D., Cakmak, M.: Complex flow and temperature history during melt extrusion in material extrusion additive manufacturing. Addit. Manuf. 22, 197 - 206 (2018). DOI https://doi.org/ 10.1016/j.addma.2018.05.015. URL https ://www.sciencedirect.com/science/article/pii/ S2214860417303718

16. Pigeonneau, F., Xu, D., Vincent, M., Agassant, J.F.: Heating and flow computations of an amorphous polymer in the liquefier of a material extrusion 3d printer. Addit. Manuf. 32, 101001 (2020). DOI 10.1016/j.addma.2019.101001

17. Seppala, J.E., Migler, K.D.: Infrared thermography of welding zones produced by polymer extrusion additive manufacturing. Addit. Manuf. 12, 71-76 (2016)

18. Serdeczny, M.P., Comminal, R., Pedersen, D.B., Spangenberg, J.: Experimental validation of a numerical model for the strand shape in material extrusion additive manufacturing. Addit. Manuf. 24, 145 - 153 (2018). DOI https://doi.org/10.1016/j.addma.2018.09.022. URL http://www.sciencedirect.com/ science/article/pii/S2214860418304585

19. Shah, R.K., London, A.L.: Laminar flow forced convection in ducts. A Source book for compact heat exchanger analytical data. Academic Press, New York (1978)

20. Sun, Q., Rizvi, G.M., Bellehumeur, C.T., Gu, P.: Effect of processing conditions on the bonding quality of FDM polymer filaments. Rapid Prototyping J. 14(2), 72-80 (2008)

21. Thomas, J.P., Rodríguez, J.F.: Modeling the fracture strength between fused-deposition extruded roads 16. In: 2000 International Solid Freeform Fabrication Symposium, pp. 16-23 (2000)

22. Turner, B.N., Strong, R., Gold, S.A.: A review of melt extrusion additive manufacturing processes: I. Process design and modeling. Rapid Prototyping J. 20(3), 192-204 (2014). DOI 10.1108/rpj-01-2013-0012. URL http://dx.doi.org/10.1108/RPJ-01-2013-0012

23. Yang, F., Pitchumani, R.: Healing of thermoplastic polymers at an interface under nonisothermal conditions. Macromolecules 35(8), 3213-3224 (2002)

24. Zhang, Y., Guillemot, G., Bernacki, M., Bellet, M.: Macroscopic thermal finite element modeling of additive metal manufacturing by selective laser melting process. Comput. Methods Appl. Mech. Eng. 331, 514 - 535 (2018). DOI https://doi.org/10.1016/j.cma.2017.12.003. URL http://www . sciencedirect.com/science/article/pii/S0045782517307545

25. Zhang, Y., Shapiro, V.: Linear-time thermal simulation of As-Manufactured FDM components. Tech. Rep. MANU-17-1517, University of Wisconsin-Madison (2017) 